\title{
On non-uniform strains in the structure of diluted magnetic semiconductors containing Jahn-Teller $3 d$-ions
}

\author{
E. N. Maksimova, V.I. Maksimov ${ }^{\dagger}$ T.P. Surkova \\ †kokailo@rambler.ru
}

Mikheev Institute of Metal Physics, Ural Branch of RAS, 18 S. Kovalevskaya str., Yekaterinburg, 620108, Russia

\begin{abstract}
Doping of $A^{\mathrm{II}} B^{\mathrm{VI}}$ semiconductor matrices by $3 d$-ions possessing a non-spherical symmetry of $3 d$ electron shells even in small concentrations causes the Jahn-Teller effect and results in a strong destabilization of the initial crystal structure. In the present work, diffuse scattering of thermal neutrons in the vicinity of intensive Bragg reflections at $T=300 \mathrm{~K}$ are studied in details on bulk cubic crystal matrices of binary compounds - diluted magnetic semiconductors $\mathrm{Zn}_{0.9} \mathrm{Ni}_{0.1} \mathrm{~S}, \mathrm{Zn}_{0.95} \mathrm{Fe}_{0.05} \mathrm{Se}$, and $\mathrm{Zn}_{0.99} \mathrm{~V}_{0.01} \mathrm{Se}$. The data of neutronographic measurements of structure reflections scanned in tangential directions are analyzed in terms of local deteriorations of the structure arising from local non-uniform damages of the initial crystal lattice. It is shown that the neutron diffuse scattering patterns from single crystals of zinc chalcogenides heavily doped by a $3 d$-impurity reflect the statistics, characteristics of which determine the directions of polarization caused by disordered shear atomic displacements. Taking into account the long-range character of electronic-type deformation produced by foreign $3 d$-ions in the semiconductor matrice, a comparison of the presented data to neutronographic results obtained on crystals of the same binary compounds at substantially smaller levels of doping allows one to conclude that the crystallographic anisotropy of dimensions of distorted nanovolumes observed at small amounts of the destabilizing impurity changes to the polarization anisotropy of displacements at the increasing impurity concentration. The results of fitting of neutron diffuse scattering patterns obtained from zinc-chalcogenide crystals heavily doped by $3 d$-ions generating additional destructive effects in the initial instable real structure of "pure" binary semiconductors become the characteristics of damage degree of crystallographic planes of initial lattice.
\end{abstract}

Keywords: single crystals, diluted magnetic semiconductors, $3 d$-ions, real structure, neutronography.

УДК: $538.911+537.9$

\section{О неоднородных деформациях в структуре разбавленных магнитных полупроводников, содержащих ян-теллеровские $3 d$-ионы}

\author{
Максимова Е. Н., Максимов В. И. ${ }^{\dagger}$, Суркова Т.П. \\ Институт физики металлов имени М.Н. Михеева УрО РАН, ул. С. Ковалевской 18, Екатеринбург, 620108, Россия
}

Легирование полупроводниковых матриц на основе соединений $A^{\mathrm{II}} B^{\mathrm{VI}} 3 d$-ионами с не сферически симметричной электронной $d$ - оболочкой, продуцируя эффект Яна-Теллера, даже в малых концентрациях оказывает сильное дестабилизирующее влияние на исходную кристаллическую структуру. В представляемой работе, проведённой на объемных кубических кристаллах матриц двойных соединений - разбавленных магнитных полупроводников $\mathrm{Zn}_{0.9} \mathrm{Ni}_{0.1} \mathrm{~S}, \mathrm{Zn}_{0.95} \mathrm{Fe}_{0.05} \mathrm{Se}, \mathrm{Zn}_{0.99} \mathrm{~V}_{0.01} \mathrm{Se}$, подробно исследовано диффузное рассеяние тепловых нейтронов в окрестности интенсивных брэгговских рефлексов при $T=300 \mathrm{~K}$. Полученные данные нейтронографической съёмки структурных отражений в тангенциальных направлениях анализировались, исходя из представлений о локальном ухудшении качества структуры вследствие неоднородных повреждений исходной кристаллической решётки. Показано, что описание наблюдаемых картин диффузного рассеяния нейтронов монокристаллами халькогенидов цинка при их сильном легировании $3 d$-примесью подчиняется статистике, характеристики которой задают направления поляризации, создаваемой беспорядочными сдвиговыми атомными смещениями. Учитывая дальнодействие электронного вида деформации, привносимой чужеродными $3 d$-ионами в полупроводниковую матрицу, сравнение представленных в настоящей работе данных с результатами нейтрон-дифракционного эксперимента на кристаллах этих же двойных соединений при более низком уровне легирования позволяет заключить, что кристаллографическую анизотропию 
протяжённости повреждённых нанообъёмов, проявляющуюся при малом количестве дестабилизирующей примеси, с увеличением её содержания сменяет анизотропия поляризации смещений. Результат описания диффузного рассеяния нейтронов в кристаллах халькогенидов цинка при их сильном легировании $3 d$-ионами, привносящими дополнительное деструктивное воздействие в изначальную нестабильность реальной структуры «чистого» бинарного полупроводника, становится характеристикой степени повреждений кристаллографических плоскостей исходной решётки.

Ключевые слова: монокристаллы, разбавленные магнитные полупроводники, $3 d$-ионы, реальная структура, нейтронография.

\section{1. Введение}

Изучение свойств разбавленных магнитных полупроводников (РМП) - систем, получаемых замещением атомов исходной структуры магнитоактивными ионами [1] - преследует несколько целей. Названные объекты по праву относятся к модельным, а широта их электронно-оптических применений требует развития зонной инженерии [1 - 5]. Вопросы, касающиеся создания полупроводниковой спинтроники на основе РМП, сегодня по-прежнему остаются открытыми [6-10].

Халькогениды цинка с характерными для них структурами кубического сфалерита и гексагонального вюрцита [11] оказались наиболее популярны в качестве матриц для синтеза РМП. Особый интерес при этом представляет изучение закономерностей формирования магнитных подсистем, что в случае легирования $3 d$-ионами, за исключением $\mathrm{Mn}^{2+}$, затруднено из-за низких пределов их растворимости [11].

Существенная проблема растворимости $3 d$-ионов с несферической $d$ - оболочкой в объёмных кристаллах халькогенидов цинка, очевидно, связана с возникновением деформаций «электронного» вида, на что указывают проявления эффекта Яна-Теллера [12-13] вместе с признаками дальнодействия электронных корреляций, обуславливающих привносимые искажения [14]. Названные представления, сформулированные на основе обнаруженных экспериментальных фактов: температурных зависимостей характеристик распространения ультразвука [12], данных диффузного рассеяния нейтронов на слабо легированных кристаллах [14], объясняются изменением электронной плотности вдоль связей примесь-анион [14]. Рассматривая данные кристаллических структур соединений $3 d$-металлов с элементами группы VI, упомянутая для халькогенидов цинка проблема растворимости в подавляющем большинстве случаев сводится к известной проблеме изодиморфизма [15].

Изучение повреждений структуры достаточно продуктивно при использовании дифракционных методик [16]. В этом отношении показательны данные диффузного рассеяния нейтронов на легированных $3 d$-примесью полупроводниковых кристаллах [17 - 18], поскольку данные других методов (в частности оптических, эффективных при небольшом количестве $3 d$-ионов [19]) становится трудно интерпретировать по мере повышения содержания легирующей примеси.

В настоящей работе нами проведено нейтронографическое исследование сильно легированных кристаллов $\mathrm{Zn}_{0.9} \mathrm{Ni}_{0.1} \mathrm{~S}, \mathrm{Zn}_{0.95} \mathrm{Fe}_{0.05} \mathrm{Se}, \mathrm{Zn}_{0.99} \mathrm{~V}_{0.01} \mathrm{Se}$. В связи с изложенным выше, выбор объектов обусловлен интересом к изучению реальной структуры РМП при уровнях легирова- ния, близких к пределу растворимости $3 d$-ионов, с целью обнаружения коллективных деформационных эффектов (предпереходных состояний), способных указать новые пути наноструктурирования магнитоактивной примеси. Полученные в представляемой работе результаты из характеристик обнаруженного диффузного рассеяния нейтронов исследованными кристаллами, проясняют дестабилизирующее влияние $3 d$-примеси на исходную кристаллическую структуру матрицы легирования.

\section{2. Образцы и методика эксперимента}

Исследованные в работе монокристаллы $\mathrm{Zn}_{0.9} \mathrm{Ni}_{0.1} \mathrm{~S}$, $\mathrm{Zn}_{0.95} \mathrm{Fe}_{0.05} \mathrm{Se}, \mathrm{Zn}_{0.99} \mathrm{~V}_{0.01} \mathrm{Se}$ были синтезированы В. Гириатом (Венесуэлла) из газовой фазы методом химического транспорта. Они представляли собой кусочки неправильной формы с линейными размерами около $0.5 \mathrm{~cm}$ в трёх взаимно-перпендикулярных направлениях. Отработанная и проверенная технология выращивания кристаллов гарантирует, что неточность указания количества примеси в формульных единицах не превышает разряда второй значащей цифры. Нейтронографическая аттестация показала, что основной структурный мотив для всех трёх кристаллов следует считать кубическим.

Эксперименты по упругому рассеянию тепловых нейтронов были выполнены на многоканальном дифрактометре Д7б (реактор ИВВ-2М, Свердловская область, г. Заречный).

\section{3. Результаты и обсуждение}

На Рис. 1-3(a,b) приведены картины рассеяния в окрестности брэгговских рефлексов кристаллов $\mathrm{Zn}_{0.9} \mathrm{Ni}_{0.1} \mathrm{~S}, \quad \mathrm{Zn}_{0.95} \mathrm{Fe}_{0.05} \mathrm{Se}, \quad \mathrm{Zn}_{0.99} \mathrm{~V}_{0.01} \mathrm{Se}$ соответственно. Для целей сравнения на Рис. $1-3(\mathrm{c}, \mathrm{d})$ мы привели (по данным работы [18]) аналогичные картины для кристаллов селенида цинка с существенно меньшим количеством соответствующей примеси ( 0.001 в формульной единице). Узлы обратной решётки кристаллов на Рис. 1-3 сканированы в тангенциальных направлениях: (a),(c) - (022) вдоль [100] (светлые кружки) и (220) вдоль [1̄ㅣ (тёмные кружки); (b), (d) - (400) вдоль [011] (светлые кружки) и (400) вдоль [010] (тёмные кружки). Функции описания диффузного рассеяния при сильном легировании кристаллов обозначены цифрами 1, 2 и 3. На вставках - фрагменты картин в увеличенном масштабе, иллюстрирующие различия в описании диффузного вклада: сплошные линии в сечении (110), пунктир - в сечении (100). Вертикальные шкалы на картинах нормированы по пиковой интенсивности структурного рефлекса, равной 100 ед. 


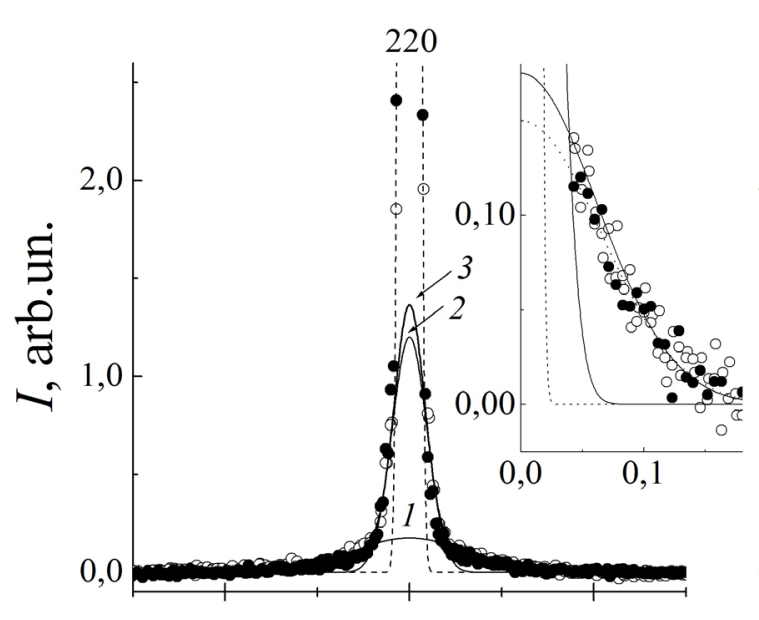

a
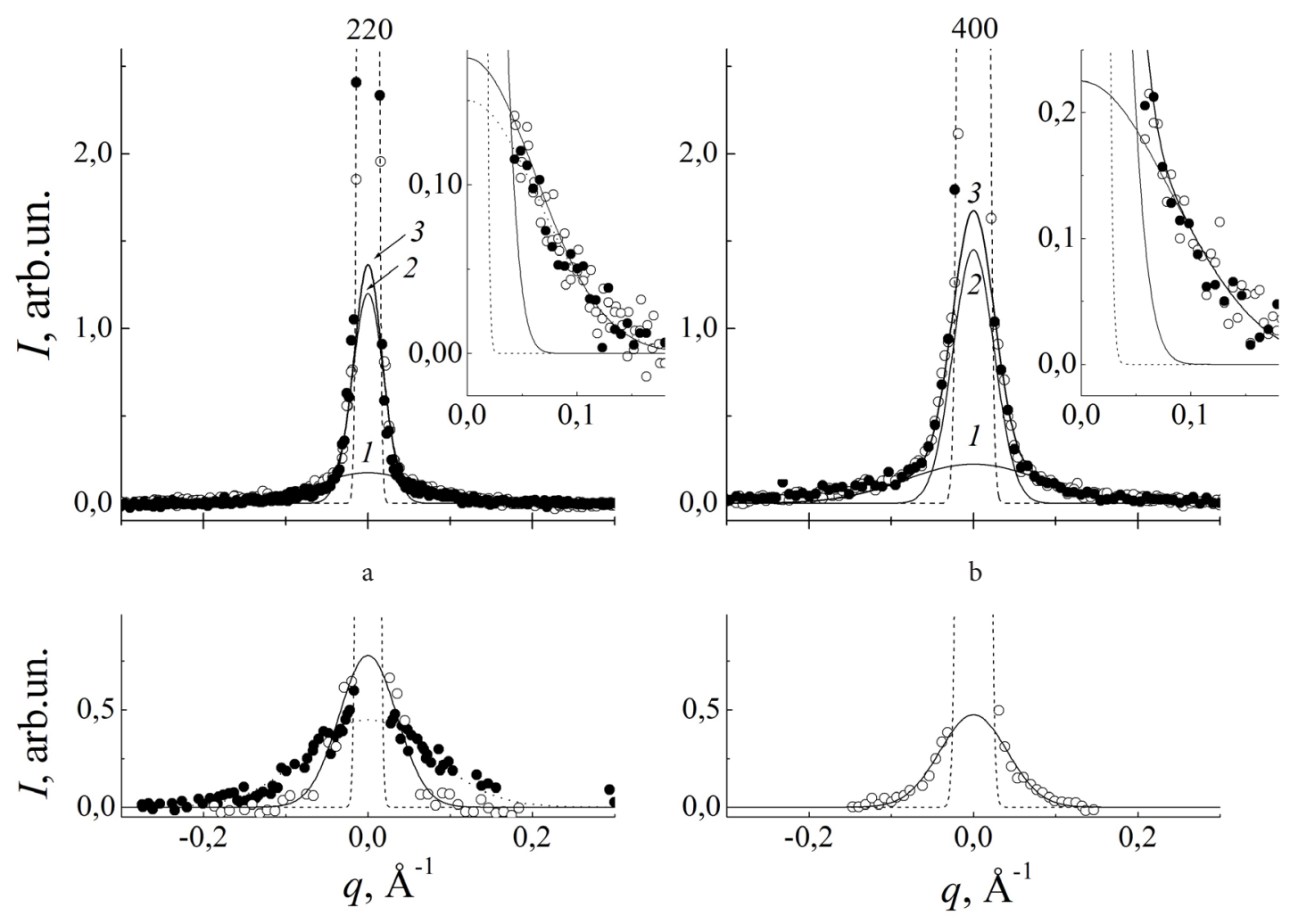

b

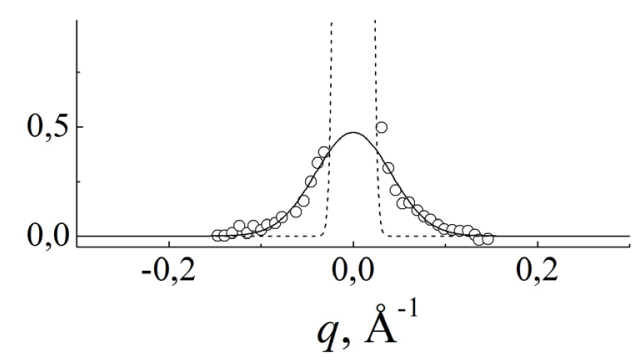

C

$\mathrm{d}$

Рис. 1.Картины рассеяния нейтронов при 300 К в окрестности брэгговских рефлексов, измеренные в тангенциальных направлениях на кристаллах $\mathrm{Zn}_{0.9} \mathrm{Ni}_{0.1} \mathrm{~S}$ (a, b) и $\mathrm{Zn}_{0.997} \mathrm{Ni}_{0.003} \mathrm{Se}(\mathrm{c}, \mathrm{d})$.

Fig. 1. Neutron diffraction scans at $T=300 \mathrm{~K}$ in the vicinity of the Bragg reflections measured along tangential directions on $\mathrm{Zn}_{0.9} \mathrm{Ni}_{0.1} \mathrm{~S}(\mathrm{a}, \mathrm{b}$ ) and $\mathrm{Zn}_{0.997} \mathrm{Ni}_{0.003} \mathrm{Se}(\mathrm{c}, \mathrm{d})$ crystals.
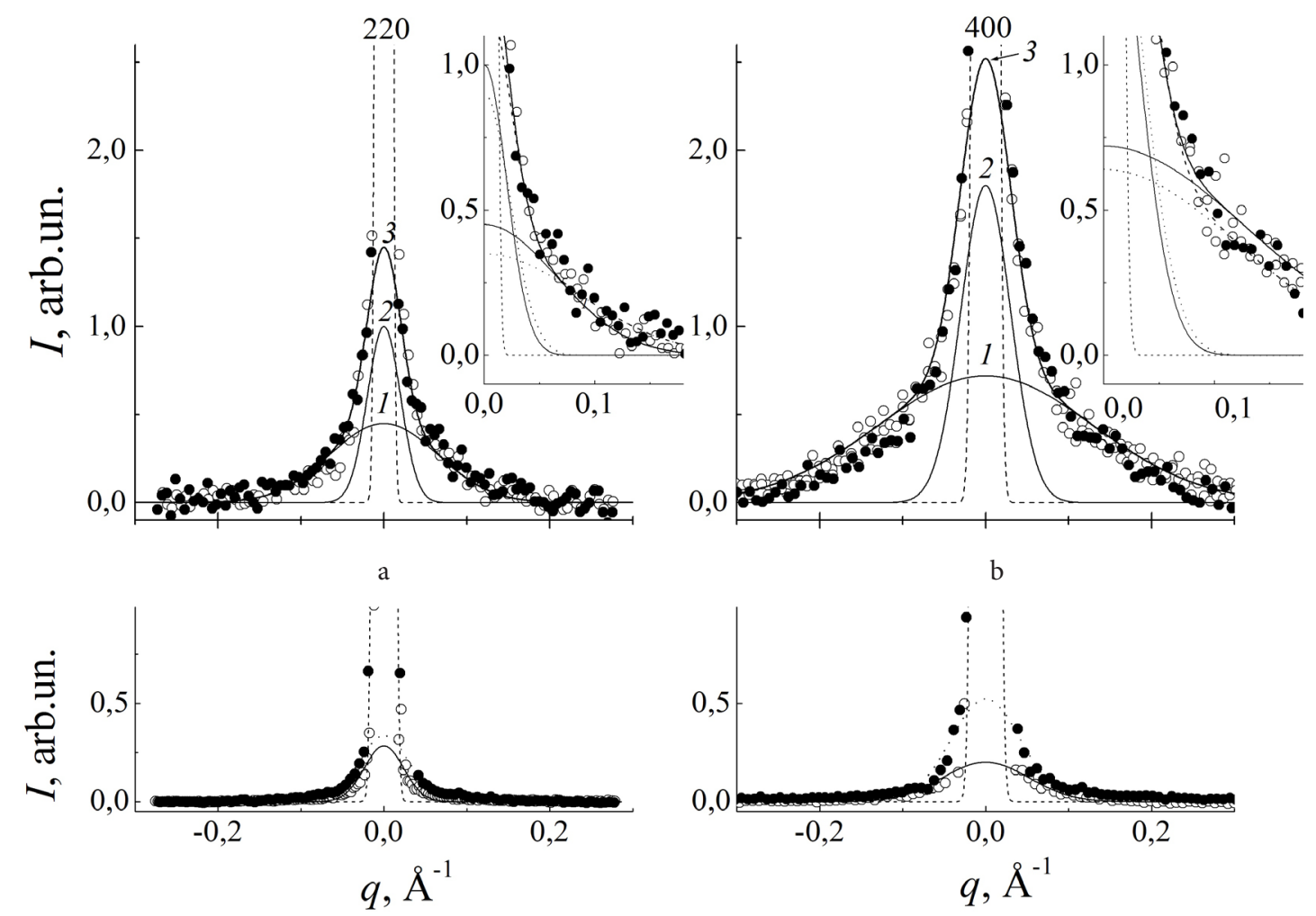

c

d

Рис. 2. Картины рассеяния нейтронов при 300 К в окрестности брэгговских рефлексов, измеренные в тангенциальных направлениях на кристаллах $\mathrm{Zn}_{0.95} \mathrm{Fe}_{0.05} \mathrm{Se}(\mathrm{a}, \mathrm{b})$ и $\mathrm{Zn}_{0.999} \mathrm{Fe}_{0.001} \mathrm{Se}(\mathrm{c}, \mathrm{d})$.

Fig. 2. Neutron diffraction scans at $T=300 \mathrm{~K}$ in the vicinity of the Bragg reflections measured along tangential directions on $\mathrm{Zn}_{0.95} \mathrm{Fe}_{0.05} \mathrm{Se}(\mathrm{a}, \mathrm{b})$ and $\mathrm{Zn}_{0.999} \mathrm{Fe}_{0.001} \mathrm{Se}(\mathrm{c}, \mathrm{d})$ crystals. 


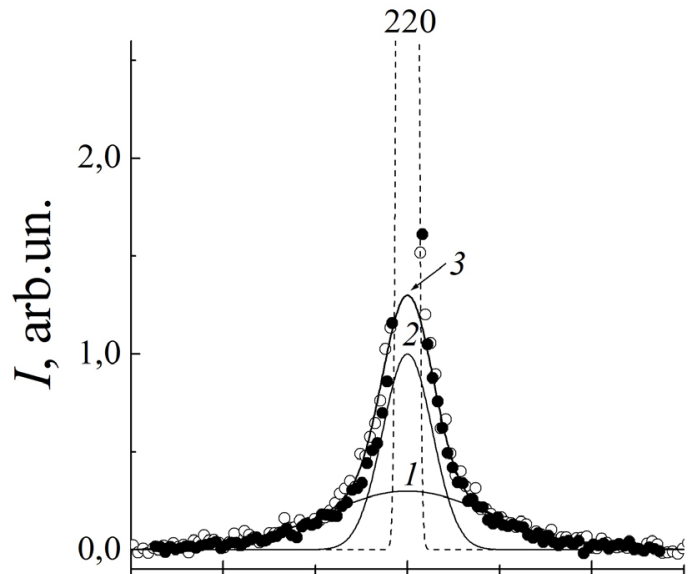

a

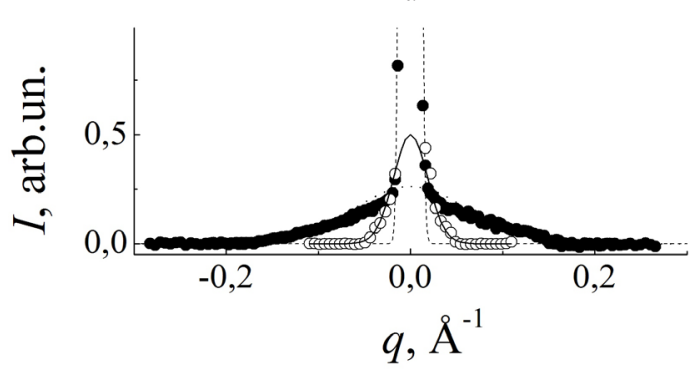

C

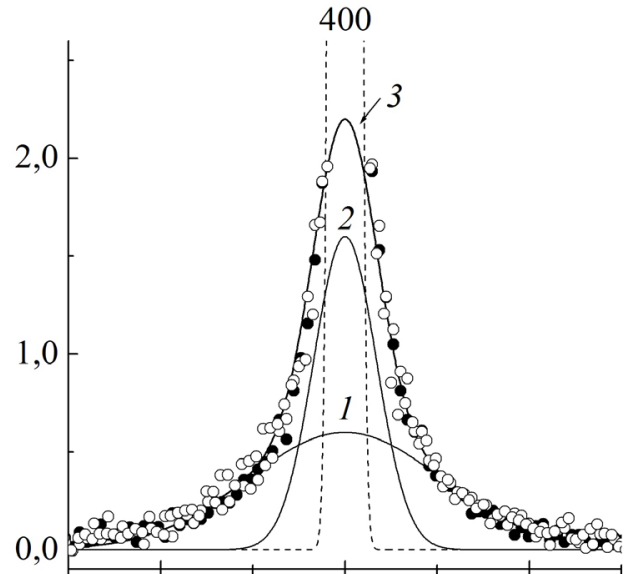

b

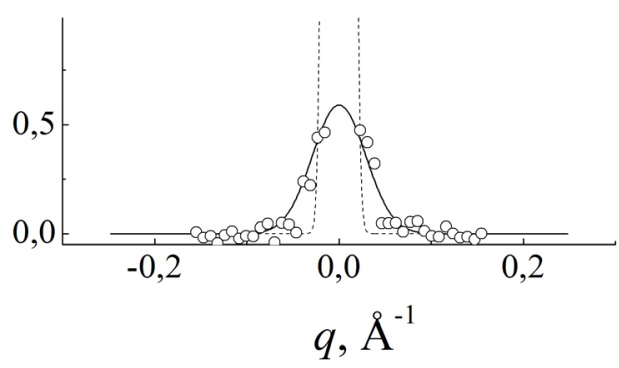

d

Рис. 3. Картины рассеяния нейтронов при 300 К в окрестности брэгговских рефлексов, измеренные в тангенциальных направлениях на кристаллах $\mathrm{Zn}_{0.99} \mathrm{~V}_{0.01} \mathrm{Se}(\mathrm{a}, \mathrm{b})$ и $\mathrm{Zn}_{0.998} \mathrm{~V}_{0.002} \mathrm{Se}$ (c, d).

Fig. 3. Neutron diffraction scans at $T=300 \mathrm{~K}$ in the vicinity of the Bragg reflections measured along tangential directions on $\mathrm{Zn}_{0.99} \mathrm{~V}_{0.01} \mathrm{Se}_{(\mathrm{a}, \mathrm{b})}$ and $\mathrm{Zn}_{0.998} \mathrm{~V}_{0.002} \mathrm{Se}$ (c, d) crystals.

Результаты проанализированы в предположении, что наблюдаемый диффузный вклад обусловлен ухудшением качества структуры кристаллов на локальном уровне. Из картин нейтронного рассеяния важны два параметра диффузного максимума: его полуширина $\Delta q$, связанная со средним размером структурной неоднородности, и высота $h$, пропорциональная квадрату средней величины смещений ионов и их концентрации [17].

В экспериментальной ситуации при низком уровне катионного замещения $3 d$-ионами в кристалле ZnSe набор корреляционных длин указывает на формирующиеся структурные неоднородности - нанообъёмы эллипсоидальной формы. С понижением температуры эти эллипсоиды разрастаются и ориентируются вдоль кристаллографических направлений, выявляя два типа искажений - тетрагональный (при легировании ионами $\mathrm{Fe}, \mathrm{Cr}$ ) и тригональный (в случае $\mathrm{V}, \mathrm{Ni})$ [18].

Габариты функций, описывающих диффузное рассеяние сильно легированных кристаллов, существенно возрастают. Если при малых концентрациях примеси диффузный вклад в окрестностях брэгговских рефлексов удовлетворительно описывается одной функцией Гаусса, для случая сильного легирования к описанию наиболее подходит сумма двух функций Гаусса (обозначенная цифрой 3 на Рис. 1 - 3). Физический смысл полученного описания подразумевает два типа деформированных микрообластей:
1) одиночные изолированные области, общее количество которых должно быть мало в объёме образца (кривые 1 на верхних панелях Рис. 1-3);

2) микрообласти, сформированные перекрытием нескольких областей первого типа (кривые 2 на верхних панелях Рис. 1 -3).

Важной особенностью диффузного рассеяния исследованных нами кристаллов является то, что его интенсивность определяется принадлежностью отражающей плоскости семействам, определяемым кубической симметрией, в пределах конкретного набора индексов Миллера. Если характеризовать сдвиговые смещения каждого атома относительно его положения в идеальной исходной решётке полярным вектором, высоту диффузного максимума определяет направление вектора поляризации. Анизотропию протяжённости неоднородных областей атомных смещений сменяет создаваемая ими анизотропия поляризации.

Полученный результат заставляет по-новому взглянуть на понимание диффузного рассеяния нейтронов в кристаллах с высоким содержанием ян-теллеровских ионов. Повреждённость структуры вследствие сдвиговых атомных смещений описывает статистика, характеристики которой задают направления создаваемой ими поляризации. Данные, отражающие выявленную статистику, приведены в Табл. 1. Наблюдаемые эффекты диффузного рассеяния в случае сильного легирования отражают степень повреждения кристаллографических плоскостей. 
Табл. 1. Статистическая зависимость корреляционных длин (протяжённостей неоднородных областей по типам 1 и 2) и интенсивностей диффузных максимумов от направления поляризации смещений из данных нейтронографических измерений структурных пиков в тангенциальных направлениях. Интенсивности диффузных максимумов $I_{m}$ даны в процентном отношении к соответствующим максимумам структурных пиков.

Table 1. Statistic dependencies of correlation lengths (elongations of unhomogeneous regions on types 1 and 2) and diffuse maxima intensities on polarization directions of displacements from neutronographic measurements data of structure peaks along tangential directions. The intensities of diffuse maxima $I_{m}$ are given in the percents to appropriate maxima of the structure peaks

\begin{tabular}{|c|c|c|c|c|}
\hline $\begin{array}{c}\text { Кристалл } \\
\text { Crystal }\end{array}$ & $\begin{array}{c}\text { Направление поляризации } \\
\text { смещений } \\
\text { The polarization direction of } \\
\text { displacements }\end{array}$ & $\begin{array}{c}\text { Корреляционная длина } \\
L_{1}^{*}, \text { нм } \\
\text { The correlation length } \\
L_{1}, \mathrm{~nm}\end{array}$ & $\begin{array}{c}\text { Корреляционная длина } \\
L_{2}^{*}, \text { нм } \\
\text { The correlation length } \\
L_{2}, \mathrm{~nm}\end{array}$ & $I_{m}, \%$ \\
\hline $\mathrm{Zn}_{0.9} \mathrm{Ni}_{0.1} \mathrm{~S}$ & $\begin{array}{l}<110> \\
<100>\end{array}$ & $\begin{array}{l}4.4 \pm 0.1 \\
3.2 \pm 0.1\end{array}$ & $\begin{array}{c}13 \pm 2 \\
9 \pm 1\end{array}$ & $\begin{array}{l}\sim 1.4 \\
\sim 1.9\end{array}$ \\
\hline $\mathrm{Zn}_{0.95} \mathrm{Fe}_{0.05} \mathrm{Se}$ & $\begin{array}{l}<110> \\
<100>\end{array}$ & $\begin{array}{l}4.0 \pm 0.5 \\
2.2 \pm 0.1\end{array}$ & $\begin{array}{l}12 \pm 1.5 \\
8.5 \pm 0.5\end{array}$ & $\begin{array}{l}\sim 1.5 \\
\sim 2.5\end{array}$ \\
\hline $\mathrm{Zn}_{0.99} \mathrm{~V}_{0.01} \mathrm{Se}$ & $\begin{array}{l}<110> \\
<100>\end{array}$ & $\begin{array}{l}3.8 \pm 0.3 \\
2.8 \pm 0.3\end{array}$ & $\begin{array}{l}9.8 \pm 0.3 \\
7.3 \pm 0.3\end{array}$ & $\begin{array}{l}\sim 1.3 \\
\sim 2.1\end{array}$ \\
\hline
\end{tabular}

Примечание ${ }^{*}$ : значения физических величин $L_{1,2}$ в колонках записаны в форме $\langle L\rangle \pm\langle\Delta L\rangle$, где $\langle L\rangle-$ математическое ожидание, $<\Delta L>-$ стандартное отклонение.

Наблюдению диффузного рассеяния нейтронов на кристаллах халькогенидов цинка может мешать существенное проявление блочности. В частности, на кристаллах системы $\mathrm{Zn}_{1-x} \mathrm{~V}_{x} \mathrm{Se}$ с содержанием $x=0.05$ мы не смогли корректно обработать данные [20].

Видится справедливым, что в случае сильного легирования будет преобладать не «динамическая» «электронная» составляющая беспорядка, а статическая или «геометрическая» деформация. Иначе, «отклик» матрицы на существенное повышение уровня легирования следует искать в изменениях её сети дефектов.

\section{4. Выводы}

В настоящей работе проведено нейтронографическое исследование кристаллов $\mathrm{Zn}_{0.9} \mathrm{Ni}_{0.1} \mathrm{~S}, \mathrm{Zn}_{0.95} \mathrm{Fe}_{0.05} \mathrm{Se}$, $\mathrm{Zn}_{0.99} \mathrm{~V}_{0.01} \mathrm{Se}$ и проанализирован диффузный вклад, измеренный в тангенциальных направлениях в окрестности основных брэгговских рефлексов. Впервые показано, что диффузное рассеяние в окрестности структурных отражений исследованных кристаллов, проявляя анизотропию поляризации смещений, становится характеристикой степени повреждения кристаллографических плоскостей. Для исследованного эффекта получена описательная статистика.

Благодарности/Acknowledgements. Paбота выполнена с использованием УНУ «НМК ИФМ» в рамках государственного задания ФАНО России по теме «Поток» № AAAA-A18-118020190112-8./The research was carried out at IMP Neutron Material Science Complex within the state assignment of FASO of Russia (theme "Flux" No. AAAA-A18-118020190112-8).

\section{Литература/References}

1. J. Kossut, J.A. Gaj. (Eds.) Introduction to the Physics of Diluted Magnetic Semiconductors. Springer series in material sci., 144. Springer (2010) 469 p.

2. M.D. McCluskey, E.E. Haller. Dopants and Defects in Semiconductors. CRC Press, Taylor \& Francis Group, Bosca Raton, London, New-York (2018) 350 p.

3. N. Myoung, V.V. Fedorov, S. B. Mirov, L.E. Wenger. J. of Luminesc. 132, 600 (2012). DOI: 10.1016/j.jlumin.2011.10.009

4. K. S. Lee, G. Oh, E. K. Kim. Solar Energy 164, 262 (2018). DOI: 10.1016/j.solener.2018.02.074

5. S. Mirov, I. Moskalev, S. Vasilyev, V. Smolski, V. Fedorov, D. Martyshkin, J. Peppers, M. Mirov, A. Dergachev, V. Gapontsev. IEEE Journal of Selected Topics in Quantum Electronics, 2018, accepted for publication. DOI: 10.1109/JSTQE.2018.2808284

6. M. Makkar, R. Viswanatha. Current Science. 112, 1421 (2017). DOI: 10.18520/cs/v112/i07/1421-1429

7. M.R. Begam, N.M. Rao, S. Kaleemulla, N.S. Krishna, M. Kuppan, G. Krishnaiah, J. Subrahmanyam. Mat. Sci. in Semicond. Proc. 18, 146 (2014). DOI: 10.1016/j.mssp.2013.11.017

8. D. Saikia, R.D. Raland, J.P. Borah. Phys.E: Lowdimens. Syst. and Nanostruct. 83, 56 (2016). DOI: $10.1016 /$ j.physe.2016.04.016

9. M. Hassan, S. Younas, F. Sher, S.S. Husain, S. Riaz, S. Naseem. Appl. Phys. A. 123, 352 (2017). DOI: 10.1007/s00339-017-0975-5

10. Y.-T. Liu, L.-P. Hou, S.-Y. Zou, L. Zhang, B.-B. Liang, Y.-C. Guo, A. Bukhtiar, M.U. Farooq, B.-S.Zou. Chin. Phys. Lett. 35, 037801 (2018). DOI: $10.1088 / 0256-307 \mathrm{X} / 35 / 3 / 037801$ 
11. J. Furduna, J. Kosut. (Eds.) Semimagnetic Semiconductors. Academic Press, New-York (1988) 496p.

12. V. Gudkov, A. Lonchakov, V. Sokolov, I. Zhevstovskikh. J. Korean Phys.Soc. 53, 63 (2008). DOI:10.3938/jkps.53.63

13. V. V. Gudkov, I. B. Bersuker. In: Vibronic Interactions and the Jahn-Teller Effect: Theory and Applications. (M. Atanasovet.al.eds). Progress in Theoretical Chemistry and Physics 23. Springer Science + Business Media B. V. (2012). P.143 - 161. DOI: 10.1007/978-94-007-2384-97

14. S.F. Dubinin, V.I. Sokolov, S.G. Teploukhov, V.D. Parkhomenko, V.V. Gudkov, A.T. Lonchakov, I. V. Zhevstovskikh, N.B. Gruzdev. Phys. of the Solid State. 49, 1235 (2007). (in Russian) [С.Ф. Дубинин, В.И. Соколов, С.Г. Теплоухов, В.Д. Пархоменко, В.В. Гудков, А.Т. Лончаков, И.В. Жевстовских,

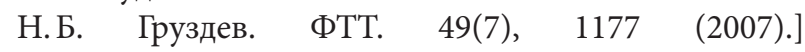
DOI: $10.1134 / \mathrm{S} 1063783407070062$

15. V.S. Urusov, N.N. Eremin. Kristallokhimija. Kratkii kurs. Ch.2. Moscow, Izd-vo Moskovskogo Universiteta (2005) 125 p. (in Russian) [В. С. Урусов, Н.Н. Еремин. Кристаллохимия. Краткий курс. Ч.2. Москва, Издво Московского университета (2005) 125 с.]
16. A.A. Rempel, A.I. Gusev. Nestehiometrija v tverdom tele. Moskva, Fizmatlit (2018) 640 p. (in Russian) [А.А. Ремпель, А.И. Гусев. Нестехиометрия в твёрдом теле. Москва, Физматлит (2018) 640 с.]

17. S.F. Dubinin, V.I. Sokolov, S.G. Teploukhov, V.D. Parkhomenko, N.B. Gruzdev. Phys. of the Solid State. 48, 2275 (2006). (in Russian) [С.Ф. Дубинин, В.И. Соколов, С.Г. Теплоухов, В. Д. Пархоменко, Н. Б. Груздев. ФТТ. 48, 2151 (2006).] DOI: $10.1134 /$ S1063783406120067

18. V.I. Maksimov, S.F. Dubinin, V.D. Parkhomenko. J. of Surface Investigation 7, 105 (2013). (in Russian) [В. И Максимов, С.Ф. Дубинин, В.Д. Пархоменко. Поверхность. 2, 9 (2013).] DOI: $10.1134 /$ S1027451013010278

19. V.F. Agekyan. Physics of the Solid State. 44, 2013 (2002). (in Russian) [В. Ф. Агекян. ФТТ. 44(11), 1921 (2002).] DOI: $10.1134 / 1.1521450$

20. V.I. Maksimov, E. N. Maksimova, T.P. Surkova. Physics of the Solid State. 60, 49 (2018). (in Russian) [В. И. Максимов, Е. Н. Максимова, Т. П. Суркова. ФТТ. 60(1), 50 (2018).] DOI: 10.1134/S1063783418010122 